\title{
ON CLOSURE PROBLEMS AND THE ZEROS OF THE RIEMANN ZETA FUNCTION'
}

\author{
NORMAN LEVINSON
}

1. In the memoirs of Wiener [7] on Tauberian theorems it is pointed out that the closure of the translations in $L(-\infty, \infty)$ of

$$
e^{(\sigma-1) x} \frac{d}{d x}\left(\frac{e^{x}}{e^{\infty x}-1}\right)
$$

is a necessary and sufficient condition for the Riemann zeta function $\zeta(s)$ to have no zeros on the line $\operatorname{Re} s=\sigma, 0<\sigma<1$.

Salem [4] using

$$
\left(1-2^{1-s}\right) \zeta(s)=\sum_{n=1}^{\infty} \frac{(-1)^{n-1}}{n^{s}}
$$

in place of $\zeta(s)$ shows that another necessary and sufficient condition is that, if $f(x)$ is a bounded measurable function on $(0, \infty)$, then

$$
\int_{0}^{\infty} \frac{x^{\sigma-1}}{e^{a x}+1} f(x) d x=0
$$

for all $a(0<a<\infty)$ should imply that $f$ is zero almost everywhere.

Here somewhat different conditions will be considered.

THEOREM I. Let $\lambda_{n}$ be a positive increasing sequence such that

$$
\sum^{\infty} \frac{1}{\lambda_{n}}=\infty \text {. }
$$

$A$ necessary and sufficient condition that $\zeta(s)$ have no zeros in the strip $\sigma_{1}<\operatorname{Re} s<\sigma_{2}$, where $1 / 2 \leqq \sigma_{1}<\sigma_{2} \leqq 1$, is that given any $\epsilon>0$ and $\alpha$ and $\beta$ such that $\sigma_{1}<\alpha<\beta<\sigma_{2}$ there exists an integer $N$ and $\left\{a_{n}\right\}$, $n=1, \cdots, N$, (depending on $\epsilon, \alpha$ and $\beta$ ) such that

$$
\int_{0}^{\infty}\left(\sum_{1}^{N} a_{n} \frac{e^{-\lambda_{n} x}}{1+e^{-\lambda_{n} x}}-e^{-x}\right)^{2}\left(x^{2 \alpha-1}+x^{2 \beta-1}\right) d x<\epsilon .
$$

A particular case of the above is with $\lambda_{n}=n$.

REMARK. It is rather trivial to show that if $\left(x^{2 \alpha-1}+x^{2 \beta-1}\right)$ in (1.1)

Received by the editors September 1, 1955 and, in revised form, October 24, 1955. 1 Sponsored in part by the Office of Naval Research. 
is replaced by $x^{2 c-1}$ for any $c, 1 / 2 \leqq c \leqq 1$, then the left side of (1.1) can always be made less than $\epsilon$ regardless of the location of zeros of $\zeta(s)$. (See end of paper.)

A result equivalent to Theorem $I$ is the following.

TheOREM II. A necessary and sufficient condition that $\zeta(s)$ have no zeros in the strip $\sigma_{1}<\mathrm{Rl} s<\sigma_{2}$ is that for any $f(x) \in L^{2}(0, \infty)$ and $\alpha$ and $\beta$ such that $\sigma_{1}<\alpha<\beta<\sigma_{2}$,

$$
\int_{0}^{\infty} \frac{e^{-\lambda_{n} x}}{1+e^{-\lambda_{n} x}}\left(x^{\alpha-1 / 2}+x^{\beta-1 / 2}\right) f(x) d x=0, \quad n=1,2, \cdots
$$

implies that $f(x)$ is zero almost everywhere on $(0, \infty)$. Here $\lambda_{n}$ satisfies (1.0) and $1 / 2 \leqq \sigma_{1}<\sigma_{2} \leqq 1$.

An immediate consequence of Theorem I is that a sufficient condition for $\zeta(s)$ to have no zeros in the strip $\left(\sigma_{1}, \sigma_{2}\right)$ is that (1.1) hold with $\alpha=\sigma_{1}$ and $\beta=\sigma_{2}$. Similarly an immediate consequence of Theorem II is that a sufficient condition for $\zeta(s)$ to have no zeros in the strip $\left(\sigma_{1}, \sigma_{2}\right)$ is that (1.2), with $\alpha=\sigma_{1}$ and $\beta=\sigma_{2}$, should imply $f(x)$ zero almost everywhere. In the case of Theorem II this follows from the fact that

$$
\frac{x^{\alpha-1 / 2}+x^{\beta-1 / 2}}{x^{\sigma 1-1 / 2}+x^{\sigma-1 / 2}}
$$

is bounded on $(0, \infty)$ and of Theorem I from the boundedness of

$$
\left(x^{2 \alpha-1}+x^{2 \beta-1}\right) /\left(x^{2 \sigma_{1}-1}+x^{2 \sigma 2-1}\right) \text {. }
$$

It has been pointed out to the author that these results can be derived with the aid of $[1 ; 2 ; 3]$. However it appears desirable to give a self-contained derivation.

2. The proof that (1.1) is a sufficient condition for $\zeta(s)$ to have no zeros in the strip $\left(\sigma_{1}, \sigma_{2}\right)$ is simple. Indeed for $\operatorname{Re} s>0$

$$
\zeta(s)\left(1-2^{1-s}\right) \Gamma(s)=\int_{0}^{\infty} \frac{e^{-x}}{1+e^{-x}} x^{s-1} d x .
$$

Let $\zeta\left(s_{0}\right)=\zeta\left(\sigma_{0}+i t_{0}\right)=0$ where $\sigma_{1}<\sigma_{0}<\sigma_{2}$. Then by (2.0) setting $x=\lambda_{n} y$ there follows

$$
\int_{0}^{\infty} \frac{e^{-\lambda_{n} y}}{1+e^{-\lambda_{n} y}} y^{\sigma_{0}+i t_{0}-1} d y=0 .
$$

Take $c$ small enough so that $\sigma_{1}<\sigma_{0}-c<\sigma_{0}+c<\sigma_{2}$ and take $\alpha=\sigma_{0}-c$ and $\beta=\sigma_{0}+c$. Then from (1.1) 


$$
\int_{0}^{\infty}\left(\sum_{1}^{N} a_{n} \frac{e^{-\lambda_{n} y}}{1+e^{-\lambda_{n} y}}-e^{\nu}\right)^{2}\left(y^{2 \sigma \sigma-2 \sigma-1}+y^{2 \sigma 0+2 \sigma-1}\right) d y<\epsilon
$$

which gives

$$
\begin{aligned}
& \int_{0}^{1}\left(\sum_{1}^{N} a_{n} \frac{e^{-\lambda_{n} y}}{1+e^{-\lambda_{n} y}}-e^{y}\right)^{2} y^{2 \sigma 0-2 c-1} d y<\epsilon, \\
& \int_{1}^{\infty}\left(\sum_{1}^{N} a_{n} \frac{e^{-\lambda_{n} y}}{1+e^{-\lambda_{n} y}}-e^{y}\right)^{2} y^{2 \sigma 0+2 c-1} d y<\epsilon .
\end{aligned}
$$

From (2.1)

$$
-\Gamma\left(\sigma_{0}+i t_{0}\right)=\int_{0}^{\infty}\left(\sum_{1}^{N} a_{n} \frac{e^{-\lambda_{n} y}}{1+e^{-\lambda_{n} y}}-e^{-y}\right) y^{\sigma 0+i t_{0}-1} d y .
$$

Writing the integral above as an integral over $(0,1)$ plus one over $(1, \infty)$ and using the Schwartz inequality it follows from (2.2) and (2.3) that

$$
\begin{aligned}
\left|\Gamma\left(\sigma_{0}+i t_{0}\right)\right| & \leqq \epsilon^{1 / 2}\left(\int_{0}^{1} y^{2 \sigma-1} d y\right)^{1 / 2}+\epsilon^{1 / 2}\left(\int_{1}^{\infty} y^{-2 c-1} d y\right)^{1 / 2} \\
& =\left(\frac{2 \epsilon}{c}\right)^{1 / 2} .
\end{aligned}
$$

Since $\epsilon$ can be taken arbitrarily small and $\Gamma\left(\sigma_{0}+i t_{0}\right) \neq 0$ this is impossible. Thus $\zeta(s)$ cannot vanish $^{2}$ in the strip $\sigma_{1}<\mathrm{Rl} s<\sigma_{2}$.

3. Here the necessity of the condition of Theorem II will be proved; that is, it will be shown that if $\zeta(s)$ has no zeros in $\left(\sigma_{1}, \sigma_{2}\right)$ then (1.2) implies $f(x)$ is zero.

First it will be shown that (1.2) implies that if

$$
H(w)=\int_{0}^{\infty} \frac{e^{-w x}}{1+e^{-w x}}\left(x^{\alpha-1 / 2}+x^{\beta-1 / 2}\right) f(x) d x
$$

then for $\operatorname{Re} w>0$,

$$
H(w)=0 .
$$

Let $w=u+i v$. Let $c>0$. For $u \geqq c$ and $0<x<1 /|v|$

$$
\operatorname{Re}\left(1+e^{-v x}\right)=1+e^{-u x} \cos v x \geqq 1+e^{-u x} \cos 1 \geqq 1 .
$$

2 The trivial character of all such sufficiency proofs seems to indicate that if the Riemann hypothesis is true the closure theorems do not seem to be a very promising direction to pursue. 
For $x \geqq 1 /|v|$

$$
\operatorname{Re}\left(1+e^{-w x}\right) \geqq 1-e^{-u x} \geqq 1-e^{-u /|v|} \geqq 1-e^{-c /|v|} .
$$

Thus for all $x>0, u \geqq c$,

$$
\left|1+e^{-w x}\right| \geqq 1-e^{-c /|v|} \text {. }
$$

For $|v| \leqq c, \quad 1-e^{-c /|v|} \geqq 1-e^{-1}>1 / 2$ and for $|v| \geqq c, \quad 1-e^{-c /|v|}$ $\geqq c / 2|v|$. Thus for small $c$ it follows from (3.2) that

$$
\frac{1}{\left|1+e^{-v x}\right|} \leqq 2 \frac{1+|v|}{c} .
$$

Therefore the integrand for $H(w)$ satisfies

$$
\begin{aligned}
\mid \frac{e^{-v x}}{1+e^{-v x}}\left(x^{\alpha-1 / 2}+\right. & \left.x^{\beta-1 / 2}\right) f(x) \mid \\
& \leqq \frac{4}{c}(1+|v|) e^{-c x}|f(x)| \max \left(1, x^{\beta-1 / 2}\right) .
\end{aligned}
$$

Using (3.3) in (3.0) and applying the Schwartz inequality it follows that the integral for $H(w)$ is uniformly convergent for $w$ in any bounded domain in $u \geqq c$. Thus $H(w)$ is analytic for $u>c$ and since $c$ is arbitrary it follows that $H(w)$ is analytic for $u>0$. Also by the Schwartz inequality and (3.3)

$$
\begin{aligned}
|H(w)| \leqq & \frac{4}{c}(1+|w|)\left(\int_{0}^{\infty} e^{-2 c x}\left(1+x^{2 \beta-1}\right) d x\right)^{1 / 2} \\
& \cdot\left(\int_{0}^{\infty}|f(x)|^{2} d x\right)^{1 / 2} .
\end{aligned}
$$

In particular if $c=1$

$$
|H(w)| \leqq K|w|, \quad u \geqq 1,
$$

where $K$ is a constant. Applying an inequality of Carleman [6, p. 130] to $H(w)$ in the half-plane $u \geqq 1$ it follows that the sum of the reciprocals of the real zeros of $H(w)$ for $u>2$ must converge unless $H$ is zero. But by (1.0) this proves (3.1).

Lemma. For any fixed real $p$ there exists a function $R(u)$ continuous for $u>0$ and such that

$$
\int_{0}^{\infty} u^{-k}|R(u)| d u<\infty
$$


for all $k, \sigma_{1}<k<\sigma_{2}$, and

$$
\int_{0}^{\infty} \frac{e^{-u x}}{1+e^{-u x}} R(u) d u=\exp \left(-\frac{1}{2} \log ^{2} x+i p \log x\right) .
$$

The proof of this lemma will be given in $\$ 4$. Let

$$
\begin{aligned}
I & =\int_{0}^{\infty} R(u) H(u) d u \\
& =\int_{0}^{\infty} R(u) d u \int_{0}^{\infty} \frac{e^{-u x}}{1+e^{-u x}}\left(x^{\alpha-1 / 2}+x^{\beta-1 / 2}\right) f(x) d x .
\end{aligned}
$$

Using the Schwartz inequality

$$
\begin{aligned}
J & =\int_{0}^{\infty}|R(u)| d u \int_{0}^{\infty} \frac{e^{-u x}}{1+e^{-u x}} x^{\alpha-1 / 2}|f(x)| d x \\
& \leqq \int_{0}^{\infty}|R(u)| d u\left(\int_{0}^{\infty}\left(\frac{e^{-u x}}{1+e^{-u x}}\right)^{2} x^{2 \alpha-1} d x\right)^{1 / 2}\left(\int_{0}^{\infty}|f(x)|^{2} d x\right)^{1 / 2} .
\end{aligned}
$$

Since

$$
\begin{aligned}
\int_{0}^{\infty}\left(\frac{e^{-u x}}{1+e^{-u x}}\right)^{2} x^{2 \alpha-1} d x & =u^{-2 \alpha} \int_{0}^{\infty}\left(\frac{e^{-y}}{1+e^{-y}}\right)^{2} y^{2 \alpha-1} d y, \\
J & \leqq C_{1} \int_{0}^{\infty} u^{-\alpha}|R(u)| d u
\end{aligned}
$$

where $C_{1}$ is a constant. By (3.5) with $k=\alpha$ it follows that $J$ is bounded. The same proof holds with $\alpha$ replaced by $\beta$. Thus the repeated integral representing $I$ is absolutely convergent and the order of integration can be inverted. Doing this and using (3.6)

$$
I=\int_{0}^{\infty}\left(x^{\alpha-1 / 2}+x^{\beta-1 / 2}\right) f(x) \exp \left(-\frac{1}{2} \log ^{2} x+i p \log x\right) d x .
$$

Setting $x=e^{y}$

$$
I=\int_{-\infty}^{\infty} G(y) e^{i p y} d y
$$

where

$$
G(y)=\left(e^{\alpha y}+e^{\beta y}\right) f\left(e^{y}\right) e^{y / 2} e^{-y^{2} / 2} .
$$

Since $f\left(e^{y}\right) e^{y / 2} \in L^{2}(-\infty, \infty)$ it follows from the Schwartz inequality that $G(y)$ is absolutely integrable. On the other hand since $H(u)=0$ 
it follows from (3.7) that $I=0$. Since this holds for all real $p$ and since, by (3.8), $I=I(p)$ is the Fourier transform of $G(y)$ it follows that $G(y)$ is zero almost everywhere and thus $f(x)$ must be zero almost everywhere, which proves the necessity of the condition of Theorem II for $\zeta(s)$ to be free of zeros in $\left(\sigma_{1}, \sigma_{2}\right)$.

4. Here the lemma will be proved. Let

$$
R(u)=\frac{1}{i(2 \pi)^{1 / 2}} \int_{-i \infty+c}^{i \infty+c} \frac{\exp \left((s+i p)^{2} / 2\right) u^{s-1}}{\Gamma(s) \zeta(s)\left(1-2^{1-s}\right)} d s
$$

where $c$ is a constant, $\sigma_{1}<c<\sigma_{2}$. It will be shown that $R(u)$ does not depend on $c$. Indeed let $\delta>0$ and let $\sigma_{1}+\delta \leqq c \leqq \sigma_{2}-\delta$. It follows easily from familiar properties of $\zeta(s)$ [5, Theorem 9.6B] that if $\zeta(s)$ has no zeros in the strip $\sigma_{1}<\mathrm{Rl} s<\sigma_{2}$ then there is a constant $A$, which depends on $\delta$, such that if $s=\sigma+i$ then

$$
|\zeta(s)|>(2+|t|)^{-\Lambda}, \quad \sigma_{1}+\delta \leqq \sigma \leqq \sigma_{2}-\delta .
$$

Also since $1 / 2 \leqq \sigma_{1}<\sigma_{2} \leqq 1$ it follows that

$$
\left|\frac{\exp \left((s+i p)^{2} / 2\right)}{\Gamma(s)\left(1-2^{1-s}\right)}\right|<K e^{-|t|}, \quad \sigma_{1}+\delta \leqq \sigma \leqq \sigma_{2}-\delta
$$

for some $K$ which depends on $\delta$ and $p$. Thus from (4.0)

$$
|R(u)| \leqq \int_{-\infty}^{\infty} K e^{-|t|}(2+|t|)^{\Lambda} u^{c-1} d t .
$$

Or, there is a $B$ depending on $\delta$ and $p$ such that

$$
|R(u)| \leqq B u^{c-1} \text {. }
$$

That $R(u)$ does not depend on $c$ for $\sigma_{1}+\delta \leqq c \leqq \sigma_{2}-\delta$ follows at once from the Cauchy integral theorem. Since $\delta$ is arbitrary $R(u)$ does not depend on $c$ for $\sigma_{1}<c<\sigma_{2}$.

Given $k$ in (3.5) it follows from (4.3) with $c=k+\delta_{1}$ and $c=k-\delta_{1}$, for some sufficiently small $\delta_{1}>0$, that (3.5) holds.

To prove (3.6) let

$$
\begin{aligned}
F(x) & =\int_{0}^{\infty} R(u) \frac{e^{-u x}}{1+e^{-u x}} d u \\
& =\frac{1}{i(2 \pi)^{1 / 2}} \int_{0}^{\infty} \frac{e^{-u x}}{1+e^{-u x}} d u \int_{-i \infty+c}^{i \infty+c} \frac{\exp \left((s+i p)^{2} / 2\right) u^{s-1}}{\Gamma(s) \zeta(s)\left(1-2^{1-s}\right)} d s .
\end{aligned}
$$

Since the repeated integral is absolutely convergent the order may be inverted to give 


$$
F(x)=\frac{1}{i(2 \pi)^{1 / 2}} \int_{-i \infty+c}^{i \infty+o} \frac{\exp \left((s+i p)^{2} / 2\right)}{\Gamma(s) \zeta(s)\left(1-2^{1-s}\right)} d s \int_{0}^{\infty} \frac{e^{-u x}}{1+e^{-u x}} u^{s-1} d u
$$

Since

$$
\int_{0}^{\infty} \frac{e^{-u x}}{1+e^{-u x}} u^{s-1} d u=x^{-s} \Gamma(s) \zeta(s)\left(1-2^{1-s}\right)
$$

it follows that

$$
F(x)=\frac{1}{i(2 \pi)^{1 / 2}} \int_{-i \infty+c}^{i \infty 0+c}\left(\exp (s+i p)^{2} / 2\right) x^{-8} d s .
$$

Setting $s+i p=i w$ and using Cauchy's integral theorem

$$
\begin{aligned}
F(x) & =\frac{x^{i p}}{(2 \pi)^{1 / 2}} \int_{-\infty}^{\infty} e^{-w^{2} / 2} x^{-i w} d w \\
& =\frac{x^{i p}}{(2 \pi)^{1 / 2}} \int_{-\infty}^{\infty} e^{-w^{2} / 2} \exp (-i w \log x) d w=x^{i p} \exp \left(-\log { }^{2} x / 2\right)
\end{aligned}
$$

which proves (3.6).

5. If (1.2) implies that $f(x)$ is zero then (1.1) is valid. Indeed (1.2) implies that any $g(x) \in L^{2}(0, \infty)$ can be approximated arbitrarily well in $L^{2}(0, \infty)$ by the functions

$$
\frac{e^{-\lambda_{n} x}}{1+e^{-\lambda_{n} x}}\left(x^{\alpha-1 / 2}+x^{\beta-1 / 2}\right) .
$$

Thus given any $\epsilon$ there exist $N$ and $a_{n}, 1 \leqq n \leqq N$, such that

$$
\int_{0}^{\infty}\left|g(x)-\sum_{1}^{N} a_{n} \frac{e^{-\lambda_{n} x}}{1+e^{-\lambda_{n} x}}\left(x^{\alpha-1 / 2}+x^{\beta-1 / 2}\right)\right|^{2} d x<\epsilon .
$$

Let

$$
g(x)=e^{-x}\left(x^{\alpha-1 / 2}+x^{\beta-1 / 2}\right) .
$$

Then

$$
\int_{0}^{\infty}\left(e^{-x}-\sum_{1}^{N} a_{n} \frac{e^{-\lambda_{n} x}}{1+e^{-\lambda_{n} x}}\right)^{2}\left(x^{2 \alpha-1}+x^{2 \beta-1}\right) \frac{\left(x^{\alpha-1 / 2}+x^{\beta-1 / 2}\right)^{2}}{x^{2 \alpha-1}+x^{2 \beta-1}}<\epsilon .
$$

Since the numerator of the last term exceeds the denominator (1.1) follows.

Thus it is seen that if (1.2) implies $f(x)$ is zero then (1.1) holds. This in turn implies $\zeta(s)$ has no zeros in the strip $\left(\sigma_{1}, \sigma_{2}\right)$ which proves 
the sufficiency of the condition of Theorem II and completes the proof of Theorem II.

If $\zeta(s)$ has no zeros in the strip $\left(\sigma_{1}, \sigma_{2}\right)$ then (1.2) implies $f(x)$ is zero which in turn implies that (1.1) holds. Thus (1.1) is a necessary condition and this completes the proof of Theorem I.

To prove the remark at the end of Theorem I note that the closure property of the translations in $L^{2}(-\infty, \infty)$ of Wiener [7] shows that the functions

$$
x^{a-1 / 2} \frac{e^{-a x}}{1+e^{-a x}} \quad(0<a<\infty)
$$

where $c$ is fixed, $1 / 2 \leqq c \leqq 1$, are closed in $L^{2}(0, \infty)$. Using the result of $\$ 3$ based on Carleman's theorem it follows easily that

$$
x^{c-1 / 2} \frac{e^{-\lambda_{n} x}}{1+e^{-\lambda_{n} x}} \quad(n=1,2, \cdots)
$$

are closed in $L^{2}(0, \infty)$. Thus if $g(x) \in L^{2}(0, \infty)$, then given any $\epsilon>0$ there exists $N$ and $\left\{a_{n}\right\}$ such that

$$
\int_{0}^{\infty}\left(\sum_{1}^{N} a_{n} x^{c-1 / 2} \frac{e^{-\lambda_{n} x}}{1+e^{-\lambda_{n} x}}-g(x)\right)^{2} d x<\epsilon .
$$

Letting $g(x)=x^{c-1 / 2} e^{-x}$ the remark is proved.

\section{REFERENCES}

1. A. Beurling, On two problems concerning linear transformations in Hilbert space, Acta Math. vol. 81 (1949) pp. 239-253.

2. - A theorem on functions defined on a semi-group, Math. Scandinavica vol. 1 (1953) pp. 127-130. 1950.

4. R. Salem, Sur une proposition equivalente à l'hypothèse de Riemann, C.R. Acad. Sci. Paris vol. 236 (1953) pp. 1127-1128.

5. E. C. Titchmarsh, The theory of the Riemann zeta-function, Oxford, 1951.

6. - The theory of functions, Oxford, 1932.

7. N. Wiener, Tauberian theorems, Ann. of Math. vol. 33 (1932) pp. 1-100.

Massaquusetts Institute of Technology 